\title{
Role of BMI1 in epithelial ovarian cancer: investigated via the CRISPR/Cas9 system and RNA sequencing
}

\author{
Qianying Zhao ${ }^{1,2}$, Qiuhong Qian ${ }^{1,3}$, Dongyan Cao ${ }^{1}$, Jiaxin Yang ${ }^{1}$, Ting Gui ${ }^{1 *}$ and Keng Shen ${ }^{1 *}$
}

\begin{abstract}
Background: B-cell-specific Moloney murine leukemia virus integration site 1 (BMI1) might be an appropriate biomarker in the management of epithelial ovarian cancer (EOC). However, the biological role of BMI1 and its relevant molecular mechanism needs further elaboration. Clustered regularly interspaced short palindromic repeats (CRISPR)/Cas9 system is an excellent genome-editing tool and is scarcely used in EOC studies.

Methods: We first applied CRISPR/Cas9 technique to silence BMI1 in EOC cells; thereafter we accomplished various in vivo and in vitro experiments to detect biological behaviors of ovarian cancer cells, including MTT, flow cytometry, Transwell, real-time polymerase chain reaction and western blotting assays, etc.; eventually, we used RNA sequencing to reveal the underlying molecular traits driven by BMI1 in EOC.

Results: We successfully shut off the expression of BMI1 in EOC cells using CRISPR/Cas9 system, providing an ideal cellular model for investigations of target gene. Silencing BMI1 could reduce cell growth and metastasis, promote cell apoptosis, and enhance the platinum sensitivity of EOC cells. BMI1 might alter extracellular matrix structure and angiogenesis of tumor cells through regulating Focal adhesion and PI3K/AKT pathways.

Conclusion: BMI1 is a potential biomarker in EOC management, especially for tumor progression and chemo-resistance. Molecular traits, including BMI1 and core genes in Focal adhesion and PI3K/AKT pathways, might be alternatives as therapeutic targets for EOC.
\end{abstract}

Keywords: B-cell-specific Moloney murine leukemia virus integration site 1 (BMI1), Clustered regularly interspaced short palindromic repeats (CRISPR)/Cas9, Epithelial ovarian cancer (EOC), Focal adhesion pathway, PI3K/AKT pathway

\section{Background}

Epithelial ovarian cancer (EOC) is the leading cause of mortality among gynecologic cancers, and the majority of patients are diagnosed in advanced stages $[1,2]$. Approximately, $70 \%$ of the patients would relapse even if they have received optimal cytoreductive surgery (CRS) combined with standard platinum-based chemotherapy. The 5-year survival rate for advanced-stage patients is very low [3]. It has been a major research focus and challenge to search for appropriate tumor markers and effective therapeutic targets because the etiology of EOC

\footnotetext{
* Correspondence: gt_greating@sina.com; shenkeng.pumc@hotmail.com 'Department of Obstetrics and Gynecology, Peking Union Medical College Hospital, Chinese Academy of Medical Sciences and Peking Union Medical College, No.1 Shuaifuyuan, Dongcheng District, Beijing, China

Full list of author information is available at the end of the article
}

is largely unknown and the tumors present heterogeneity in multiple dimensions.

As a core member of Polycomb group proteins (PcG), Bcell-specific Moloney murine leukemia virus integration site 1 (BMI1) plays an important role in epigenetics, participates in important cellular events and is identified as aberrantly expressed in various human cancers [4-11]. In our previous study, we also found significant over-expression of BMI1 in metastatic lymph nodes and recurrent tumors compared to primary ovarian carcinomas. In addition, intensive expression of BMI1 in metastatic and recurrent tumors was an independent prognostic factor for survival and relapse, respectively [12]. However, the biological functions and related mechanism of BMI1 in EOC lack elaboration.

Among a variety of genome-editing techniques, the clustered regularly interspaced short palindromic repeats, 
(CRISPR)/Cas9 system has enormous prospects in tumor genesis and progression researches. The technique is highly effective at thoroughly shutting off gene expression with $\sim 20$ bp guide RNAs (gRNAs) [13, 14], by which can establish ideal cellular models for functional investigation. To date, studies involving the CRISPR/Cas9 technique in ovarian cancer studies are still scarce.

Therefore, we 1) explored the feasibility of the CRISPR/ Cas9 system as a genome editing tool to construct BMI1 knock-out EOC cell models, 2) investigated the changes in biological behaviors after silencing BMI1 using in vitro and in vivo experiments, and 3) identified the differences in mRNA profiles between wild-type and BMI1 knock-out EOC cells using transcriptome sequencing technique in order to reveal the potential molecular mechanism. 4) revealed the potential of BMI1 serving as a biomarker in EOC management with basic research evidences.

\section{Methods}

\section{Knocking out BMI1 in EOC cell lines using CRISPR/Cas9 technique \\ Cell culture}

The ovarian cancer cell line SKOV3 was obtained from Institute of Basic Medical Sciences Chinese Academy of
Medical Sciences \& School of Basic Medicine, Peking Union Medical College. The SKOV3 cells were cultured in McCOY' 5A medium (Macgene; Beijing, $\mathrm{CN}$ ), supplemented with $10 \%$ fetal bovine serum (FBS, HyClone; Logan, UT, US), $100 \mathrm{U} / \mathrm{ml}$ penicillin (Solarbio; Beijing, CN) , and $100 \mu \mathrm{g} / \mathrm{ml}$ streptomycin (Solarbio; Beijing, CN).

\section{CRISPR/Cas9 vector construction and transfection}

Specific gRNAs (Table 1) were designed using http:// crispr.mit.edu/, and they were subsequently connected to linear pX330-BbsI vectors. To enhance editing efficiency, two gRNAs were synthesized targeting distinct domains of BMI1. Successively, two expression vectors (pX330-Cas9gRNA1 and pX330-Cas9-gRNA2) were established, and co-transfected SKOV3 simultaneously with pLLexp-puro plasmid. Then, we used puromycin $(1 \mu \mathrm{g} / \mathrm{ml})$ to screen out those cells which have not been successfully transfected. Eventually, we cultured the viable cells surviving drugsifting in 96-well plates using limited dilution method and amplified all the single-cell derived sub-clones.

\section{Validation of BMI1 knock-out}

Genomic DNA was extracted from each sub-clone for polymerase chain reaction (PCR) and Sanger sequencing

Table 1 Sequences of gRNAs and primers

\begin{tabular}{|c|c|c|c|}
\hline \multirow[t]{2}{*}{ Name } & \multicolumn{2}{|l|}{ Sequences $\left(5^{\prime}->3^{\prime}\right)$} & \multirow[t]{2}{*}{ PAM/ Length } \\
\hline & Forward & Reverse & \\
\hline \multicolumn{4}{|c|}{ CRISPR gRNAs for BMI1 } \\
\hline gRNA1 & CACCGAACGTGTATTGTTCGTT & AAACGGTAACGAACAATACACGTTC & ACC \\
\hline gRNA2 & CACCGTGGTCTGGTCTTGTGAAC & AAACAGTTCACAAGACCAGACCAC & TGG \\
\hline \multicolumn{4}{|c|}{ Primers for $P C R$} \\
\hline $\mathrm{BMI} 1^{\mathrm{a}}$ & $\begin{array}{l}\text { TTGATGCCACAACCATAATAGAAT } \\
\text { CTAACACCAATGATTTATCCACTC }\end{array}$ & AATTACAAACAAGGAATTTCAACA & 494 bp 194 bp \\
\hline \multicolumn{4}{|c|}{ Primers for real-time PCR } \\
\hline Caspase3 & GAAATTGTGGAATTGATGCGTGA & CTACAACGATCCCCTCTGAAAAA & 166 bp \\
\hline $\mathrm{BCl}-2$ & CTAAGGGTATGAAGGACCTGTA & CTCTGGAATCTAAAGGTCGT & $111 \mathrm{bp}$ \\
\hline COL1A1 & GTGCGATGACGTGATCTGTGA & CGGTGGTTTCTTGGTCGGT & $119 \mathrm{bp}$ \\
\hline COL4A1 & GGACTACCTGGAACAAAAGGG & GCCAAGTATCTCACCTGGATCA & 240 bp \\
\hline TNC & TCCCAGTGTTCGGTGGATCT & TTGATGCGATGTGTGAAGACA & $131 \mathrm{bp}$ \\
\hline LAMA3 & TGCTCAACTACCGTTCTGCC & TCCAGTTCTTITGCGCTTTGT & 181 bp \\
\hline ITGA7 & CAGCGAGTGGACCAGATCC & CCAAAGAGGAGGTAGTGGCTATC & 203 bp \\
\hline ITGB4 & CTCCACCGAGTCAGCCTTC & CGGGTAGTCCTGTGTCCTGTA & 133 bp \\
\hline AKT3 & AATGGACAGAAGCTATCCAGGC & TGATGGGTTGTAGAGGCATCC & $130 \mathrm{bp}$ \\
\hline CREB5 & AAAGACTGCCCAATAACAGCC & AAGCTGGGACAGGACTAGCA & 88 bp \\
\hline PIK3CA & GAAACAAGACGACTTTGTGACCT & CTTCACGGTTGCCTACTGGT & $76 \mathrm{bp}$ \\
\hline PIK3CD & AGCCGGAAGACTACACGCT & GGTCAGGTGAGGGGTCAAC & 122 bp \\
\hline $\mathrm{BIRC3}$ & TTCCGTGGCTCTTATTCAAACT & GCACAGTGGTAGGAACTTCTCAT & $96 \mathrm{bp}$ \\
\hline GAPDH & ACAACTTTGGTATCGTGGAAGG & GCCATCACGCCACAGTTTC & $101 \mathrm{bp}$ \\
\hline
\end{tabular}

aLonger fragments obtained by the first BMI1 primer is more suitable for subsequent Sanger sequencing, whereas the second primer is better for revealing fragments shortening after silencing BMI1 on Agarose gel 
of the targeted fragments. PCR primers are listed in Table 1. Protein was subsequently extracted from subclones with shortened nucleic acid sequences for western blotting. All validated BMI1 knock-out clones were then amplified and stored.

\section{Comparison of biological behaviors using in vitro and in vivo experiments \\ MTT assays}

Cell viability was detected using MTT assays. Twenty microliters of MTT reagent $(5 \mathrm{mg} / \mathrm{ml})$ was added to each well (96-well plate) and incubated for $4 \mathrm{~h}$ and then terminated by adding $100 \mu$ l dimethylsulfoxide (DMSO) and incubating at $37^{\circ} \mathrm{C}$ in the dark for $10 \mathrm{~min}$. Cell proliferation was assessed by measuring the absorbance at $570 \mathrm{~nm}$ and $630 \mathrm{~nm}$ wavelength (Optical density, $\mathrm{OD}_{570-}$ $630 \mathrm{~nm})$. Growth curves were drawn with doubling time calculated for each group by consecutive MTT assays for seven days.

\section{Flow cytometry (FCM)}

The cell pattern of each sub-clone was detected using C6 FCM (BD Biosciences; New Jersey, US). Briefly, cell pretreatment comprised cold-ethanol fixation for $2 \mathrm{~h}$ and propidium iodide (PI, Keygene; Wageningen, the Netherlands) staining for $30 \mathrm{~min}$ in the dark. Modfit software (Verity; Maine, US) was used for analysis. Similarly, apoptosis could be compared by FCM after Annexin Vfluorescein isothiocyanate (FITC) and PI staining.

\section{Transwell migration and invasion assays}

The cell migration assay was performed using a Transwell chamber (BD Falcon ${ }^{\mathrm{Tm}}$, San Jose, CA). These chambers were inserted into 24-well cell culture plates. Wildtype SKOV3 cells and BMI1 knock-out clones in $200 \mu \mathrm{l}$ serum-free culture solution were added to the upper chambers. Ten percent FBS-containing McCOY' 5A medium was added into the lower chambers to serve as the chemo-attractant. For invasion assays upper chambers were pre-coated with Matrigel (BD BioCoat ${ }^{\mathrm{Tm}}, \mathrm{BD}$ Biosciences, San Jose, CA). After incubation for $24 \mathrm{~h}$, the medium, the gel and uncrossed cells in the upper chambers were removed, while the migrated/invaded cells at the lower side of the membranes were fixed with paraformaldehyde (4\%) and stained with crystal violet. Pictures were taken at 200X magnification, and cell numbers from five random microscopic fields were counted for statistical comparison.

\section{Real-time PCR and western blotting}

Expression levels of marker proteins in apoptosis were compared between wild-type and BMI1 knock-out cells by real-time PCR and western blotting. Primers and antibodies are listed in Tables 1 and 2, respectively.
Table 2 Antibodies

\begin{tabular}{lll}
\hline Name & Corporation & Dilution ratio \\
\hline BMI1 & Cell signaling & $1: 1000$ \\
Caspase3 & Abcam & $1: 1000$ \\
BCl-2 & Abcam & $1: 500$ \\
COL1A1 & Abcam & $1: 1000$ \\
COL4A1 & Bioworld & $1: 500$ \\
TNC & Abcam & $1: 2000$ \\
LAMA3 & Abcam & $1: 2000$ \\
ITGA7 & Bioworld & $1: 500$ \\
ITGB4 & Abcam & $1: 2000$ \\
AKT3 & Proteintech & $1: 500$ \\
CREB5 & Proteintech & $1: 500$ \\
PIK3CA & Abcam & $1: 1000$ \\
PIK3CD & Abcam & $1: 500$ \\
BIRC3 & Abcam & $1: 1000$ \\
$\beta$-Tubulin & Bioworld & $1: 3000$ \\
$\beta$-Actin & Cell signaling & $1: 1000$ \\
\hline
\end{tabular}

Total RNA was isolated using Trizol reagent (Invitrogen; California, US). For quantitative analysis, mRNA levels of target sequences were compared: RNA was first retro-transcribed with random primers using TransScript First-Strand cDNA Synthesis Kit (TransGen; Beijing, $\mathrm{CN}$ ), and then real-time PCR was carried out using TransStart Tip Green qPCR SuperMix (TransGen; Beijing, CN) with specific primers. The comparative $\mathrm{Ct}$ method was used to calculate the relative abundance of mRNA compared to GAPDH expression.

Harvested EOC cells were washed in phosphate buffer (PBS) and lysed in ice-cold cell lysis buffer with freshly added $0.01 \%$ protease inhibitor and then incubated on ice for $15 \mathrm{~min}$. Cell debris was discarded after ultrasonic breaking and centrifugation at $14000 \mathrm{rpm}$ for $10 \mathrm{~min}$ at $4{ }^{\circ} \mathrm{C}$. Afterwards, the supernatant was run on a sodiumdodecyl sulphate (SDS)-PAGE gel, transferred to a polyvinylidene fluoride (PVDF) membrane, hybridized with specific antibodies, and developed using electrochemiluminescence (ECL) methodology.

\section{Chemotherapeutic response}

MTT assays were also used to estimate the cells' sensitivity to cisplatin, carboplatin and paclitaxel (National Institutes for Food and Drug Control; CN). Each antineoplastic medicine was diluted at gradient concentrations and added to 96-well plates (6 wells per group per concentration). After incubation at $37{ }^{\circ} \mathrm{C}$ for $48 \mathrm{~h}, \mathrm{MTT}$ assays were performed as previously described. With $\mathrm{OD}_{570 \mathrm{~nm}}$ and $\mathrm{OD}_{630 \mathrm{~nm}}$ detected, the growth inhibition ratio was calculated under each concentration for each group (Formula 1). Afterwards, the drug concentration 
at which $50 \%$ of the cells were prevented from proliferating was obtained using GraphPad Prism 5.0 software (California, US).

Growth inhibition rate $=$

$\left(1-\frac{\text { Test well } \mathrm{OD}_{570-630 \mathrm{~nm}}-\text { Blank well OD }_{570-630 \mathrm{~nm}}}{\text { Lowest Concentration well } \mathrm{OD}_{570-630 \mathrm{~nm}}-\text { Blank well OD }_{570-630 \mathrm{~nm}}}\right) \times 100 \%$

\section{Xenografted tumor experiments}

Female nude mice (BALB/c) were purchased from Beijing Vital River Laboratory Animal Technology Corporation (Beijing, CN). All mice were housed and fed under specific pathogen-free conditions in an institution approved by the Beijing Laboratory Animal Research Center. All studies were approved and supervised by Peking Union Medical College Hospital. All mice used were 5 weeks old when the experiments initiated. Six mice were assigned per group. Cells cultivated from different groups $\left(2 \times 10^{7}\right.$ cells) were injected subcutaneously. To compare the ability of tumor formation in vivo, the transplanted tumors were checked and dimensioned every 2 days. Feeding was stopped at the same time for each group after 10 weeks. Tumor formation rate and average tumor size (Length $\times$ Width ${ }^{2} \times 0.5$ ) were calculated and compared.

\section{Investigation of underlying molecular mechanism using RNA sequencing}

Total RNA was extracted from wild-type SKOV3 and BMI1 knock-out clones, and transcriptome libraries were subsequently constructed using a KAPA stranded mRNA-Seq Kit (KAPA Biosystems; Massachusetts, US). High-throughput sequencing was accomplished on a Hiseq2000 platform (Illumina; California, US). Bioinformatics analyses included estimation of gene-expression amount, clustering, GO (Gene ontology) and KEGG (Kyoto Encyclopedia of Genes and Genome). Differential gene-expression profiles were validated by real-time quantitative PCR and western blotting; corresponding primers and antibodies are listed in Tables 1 and 2 . Eventually, potential pathways involving BMI1 in tumor genesis and progression were proposed.

\section{Statistical analysis}

Biological behaviors and transcriptome profiles were compared between wild-type and BMI1 knock-out EOC cells. Each experiment was performed in triplicate assays and repeated at least three times. All values were expressed as the means \pm SD (standard deviation). Statistical significance was determined using two-sided Student's $t$ test or Fish's exact test, and a value of $P<0.05$ was considered significant. Statistical analyses were performed using Statistical Package for the Social Sciences 20.0 (IBM; New York, US).

\section{Results}

Knock-out BMI1 in EOC cells using CRISPR/Cas9 system Specific gRNAs were inserted into pX330-Cas9 vectors as shown in Fig. 1 with a success rate of $100 \%$. After transfection and drug sifting, we obtained 23 single-cell derived BMI1 knock-out clones after limited-dilution culture. Figure 1 also depicts the validation results of target-gene editing: Agarose electrophoresis results revealed PCR products from the treated group had shortened nucleic acid sequences (9/23, Fig. 1b); target fragments of these sub-clones were confirmed deleted by Sanger sequencing (9/9); and western blotting experiments further verified that all nine sub-clones completely lack expression of BMI1 protein (Fig. 1c).

\section{Silencing BMI1 suppressed cell proliferation, migration and invasion of EOC cells}

Growth curves showed that BMI1 knocking out reduced cell proliferation straight from the second through the seventh day, compared to the wild-type SKOV3 (Fig. 2a). The doubling times for BMI1 knock-out and wild-type clones were $2.10 \pm 0.7 \mathrm{~d}$ and $5.62 \pm 1.57 \mathrm{~d}$, respectively $(P<0.05)$. BMI1 silencing also led to cell pattern alteration, as the proportion of EOC cells in S phase significantly decreased (23. $7 \%$ vs. $12.2 \%$, Fig. 2b). Cell invasiveness assessed using Matrigel-coated Transwell chambers presented a significant inhibition (Fig. 2c). Figure 2d shows reduced migration of SKOV3 cells transfected with CRISPR/Cas9-gRNAs. The numbers of migrated/invaded cells in either group are listed in Table 3. In addition, in vivo experiments (Fig. 2e) revealed that 1) tumor formation rate of subcutaneous inoculation reduced from $100 \%$ to $50 \%$ after BMI1 silencing and 2) the average xenografted tumor size was dramatically smaller in the BMI1 knock-out group $\left(5.3 \mathrm{~mm}^{3}\right)$ than in the wild-type group $\left(1986.7 \mathrm{~mm}^{3}\right)$.

\section{BMI1 knock-out promoted apoptosis of EOC cells}

We next examined the effect of BMI1 knocking out on cell apoptosis. First, FCM detected a higher apoptosis rate in BMI1 knock-out EOC cells than in the untreated group $(3.3 \pm 0.2 \%$ vs. $1.9 \pm 0.2 \%, P<0.05)$. Established markers of apoptosis include caspases. Therefore, we determined the expression of caspase 3 cleavages in control and BMI1gRNAs-transfected EOC cells. An increase of active caspase 3 was observed in BMI1 knock-out cells (Fig. 2f). Bcl-2 suppresses apoptosis in a variety of cell systems, including functioning in a feedback loop system with caspases. We detected a remarkable reduction of $\mathrm{Bcl}-2$ expression after knocking-out BMI1, indicating an ongoing apoptotic process (Fig. 2f, Additional file 1: Figure S1).

Silencing BMI1 enhanced platinum sensitivity of EOC cells We tested whether BMI1 silencing would affect chemotherapeutic responses of EOC cells. After drawing cell 


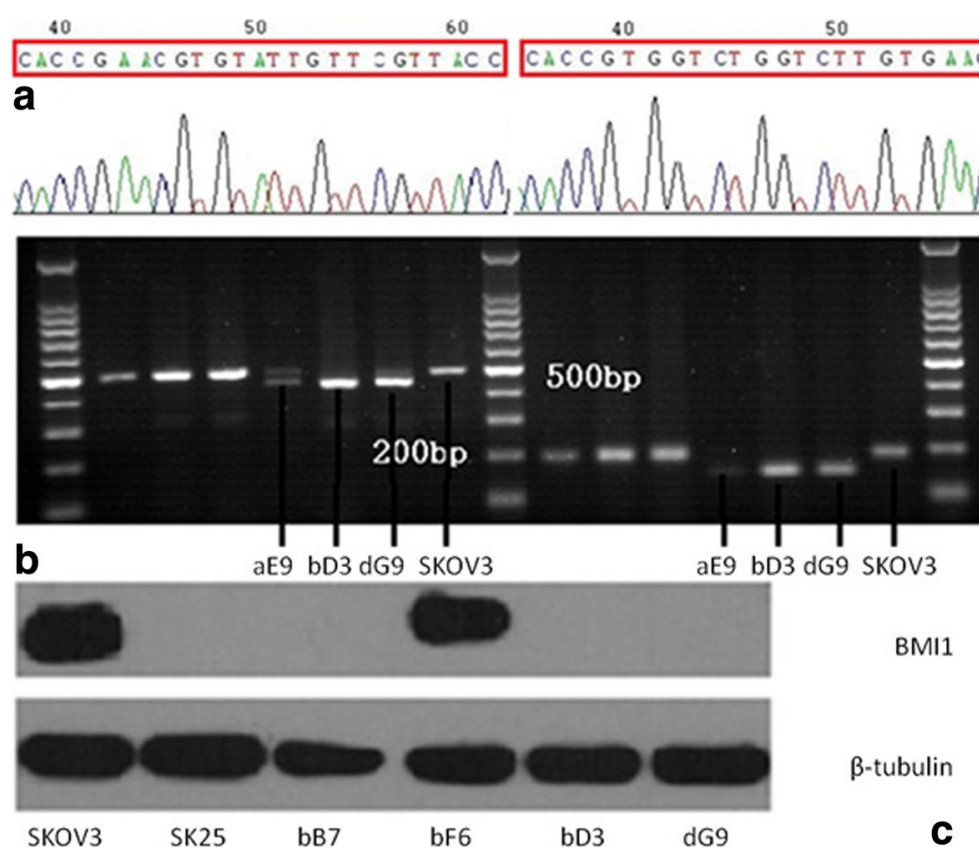

Fig. 1 a. BMI1 gRNAs successfully inserted into pX330-Bbsl vectors; $\mathbf{b}$. Sub-clones with shortened nucleic acid sequences on Agarose gel after PCR; c. Sub-clones with no expression of BMl1 protein entirely detected by western blotting

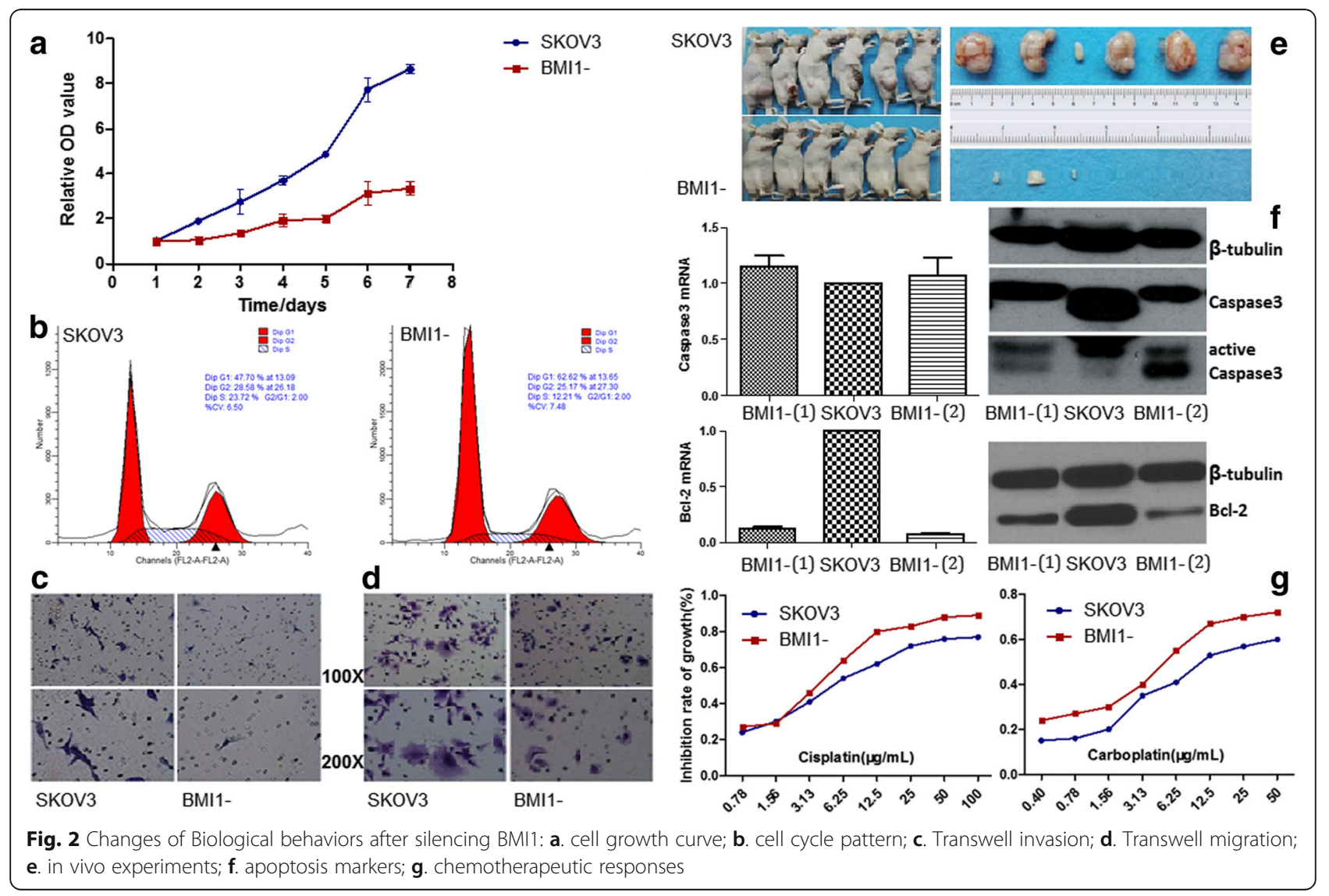


Table 3 Results of Transwell experiments and IC50 for anti-neoplastic drugs

\begin{tabular}{|c|c|c|c|c|c|}
\hline \multirow[t]{2}{*}{ Group } & \multicolumn{2}{|c|}{ Cell numbers (median \pm SD) } & \multicolumn{3}{|c|}{$\mathrm{IC50}(\mu \mathrm{g} / \mathrm{ml})$} \\
\hline & Invasion & Migration & Cisplatin & Carboplatin & Paclitaxel \\
\hline Wild-type SKOV3 & $9.2 \pm 0.4$ & $27.8 \pm 3.2$ & 5.9 & 14.5 & 16.6 \\
\hline BMI1 knock-out clone & $4.2 \pm 1.1$ & $11.0 \pm 0.7$ & 3.4 & 5.2 & 13.6 \\
\hline$P$ value & $<0.05$ & $<0.05$ & $<0.05$ & $<0.05$ & $>0.05$ \\
\hline
\end{tabular}

growth inhibition curves under gradient drug concentrations (Fig. 2g), IC50s of either sub-clone to cisplatin, carboplatin and paclitaxel were calculated (Table 3). The data demonstrated that BMI1 knock-out sensitized EOC cells to platinum medications $(P<0.05)$, including both cisplatin and carboplatin. However, similar reactions to paclitaxel were observed in BMI1-silenced and untreated cells $(P>0.05)$.

\section{BMI1 modulated focal adhesion and PI3K/AKT pathways in EOC cells}

Total RNA extracted from either group was qualified for RNA sequencing, revealing appropriate $\mathrm{OD}_{260 \mathrm{~nm} / 280 \mathrm{~nm}}$ values (1.8-2.0) and abundances ( $\geq 5.0 \mu \mathrm{g}$ per group). Raw reads were filtered and aligned to reference sequences. With GO analyses, we demonstrated that the majority of differentially expressed genes after BMI1 silencing participated in extracellular matrix (ECM) construction and blood vessel development. KEGG analyses revealed that BMI1 might alter the biological behaviors of EOC cells by modulating the focal adhesion and PI3K/AKT pathways. Expressions levels of related markers at both mRNA and protein levels were validated (Fig. 3): shutting-off BMI1 down-regulated transcription of COL1A1, COL4A1, TNC, ITGA7, ITGB4 and Bcl-2, while it up-regulated transcription of AKT3, LAMA3, CREB5 and BIRC3. Western blotting experiments on translational aspects showed results consistent with mRNA levels, except for additional under-expression of PIK3CA protein.

\section{Discussion}

The CRISPR technique traces back to 1987, when scientists discovered corresponding reverse sequences following certain DNA fragments at the terminus of a bacterial genome $[15,16]$. Between these short palindromic repeats were random DNA sequences $(\sim 30 \mathrm{bp})$, which were demonstrated later to be complementary to phage sequences [17, 18]. Recently, this technique has been generating excitement for its ability to modify genetic information rapidly and thoroughly. Compared to traditional techniques (e.g. RNA interfering, transcription activator-like effector nuclease), CRISPR/Cas9 system possesses several advantages: 1) the gRNAs are easy to design and stable in double-chain form; 2) target sequences are directly cut on DNA level, inducing an entire shut-off of gene expression; 3) multiple genes could be edited simultaneously, and DNA sequences could be knock-in as well as knock-out [13, 14]. We have successfully applied the CRISPR/Cas9 system and established a stable EOC cell model with BMI1 silenced entirely for further investigations.

BMI1 protein is one component of the polycomb repressive complex 1 (PRC1), which catalyzes lysine 119 mono-ubiquitination of histone H2A (H2AK119Ub1). H2AK119Ub1 is thought to contribute to gene silencing through the induction of chromatin compaction and inhibition of transcriptional elongation [19-21]. BMI1 has a broad impact on a diversity of cellular events: it controls the cell cycle by regulating the tumor suppressor proteins $\mathrm{p} 16^{\mathrm{INK} 4 \mathrm{a}}$ and $\mathrm{p} 14^{\mathrm{ARF}}$ [22]; it promotes cell proliferation by suppressing the $\mathrm{p} 16^{\mathrm{INK} 4 \mathrm{a}} /$ retinoblastoma and/or the p14 ARF/MDM2/p53 pathways [10]; it bypasses senescence and immortalizes cells by inducing telomerase activity in adult stem cells [9]; and it contributes to tissue homeostasis by maintaining self-renewal of hematopoietic, neural, prostate, intestinal, lung epithelial and bronchoalveolar stem cells $[5,23]$. More importantly, accumulating genetic and epigenetic evidence has revealed BMI1, serving as a cancer stem cell marker, plays a crucial role in tumor heterogeneity and relapse $[6,24]$. A growing number of recent studies have confirmed the oncogenic activation of BMI1 in diverse human malignancies and have explored the function of BMI1 as a pathway regulator in both stem cells and cancer cells $[25,26]$. We discovered a remarkable inhibition of cell proliferation from silencing BMI1, with more EOC cells staying in G1 phase. A recent study has demonstrated that the activity of the estrogen receptor $\alpha \quad(E R \alpha)$-coupled BMI1 signature impacts p16 ${ }^{\text {INK4a }}$ and cyclin D1 status and correlates with the tumor molecular subtype and biologic behavior in breast cancer [9]. In addition, knocking out BMI1 reduced both the invasion and migration abilities of EOC cells. In accordance, other studies found that BMI1 was involved in inducing epithelial mesenchymal transition, leading to tumor invasion and metastasis [26]. Moreover, overexpression of BMI1 in EOC cells was found to up-regulate the expression of cyclin D1, CDK4 and Bcl-2, promoting cell growth and inhibiting cell apoptosis [27]. In contrast, silencing BMI1 in our study manifested a reverse impact on regulation of the cell cycle and apoptosis. Last but not the least, we found that knocking out BMI1 enhanced the 

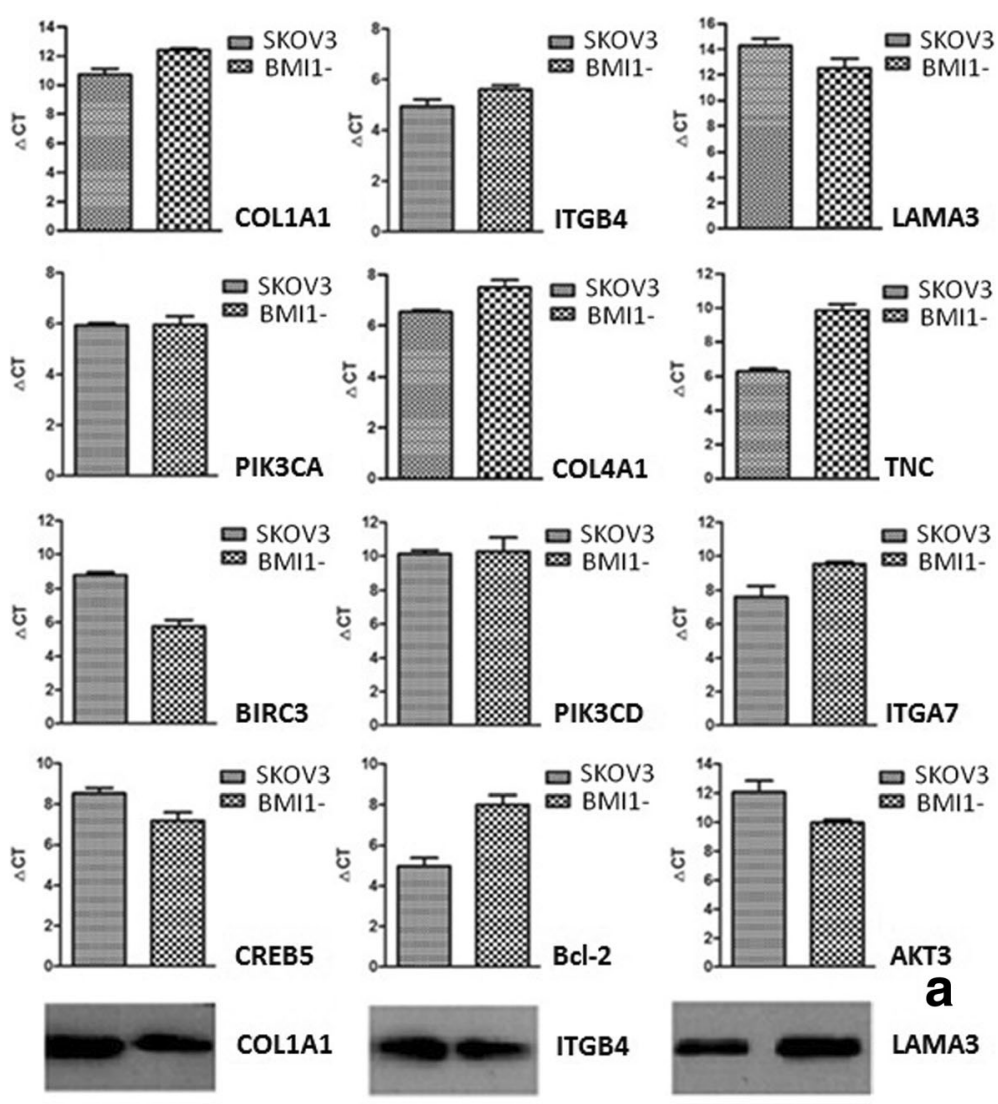

COL1A1
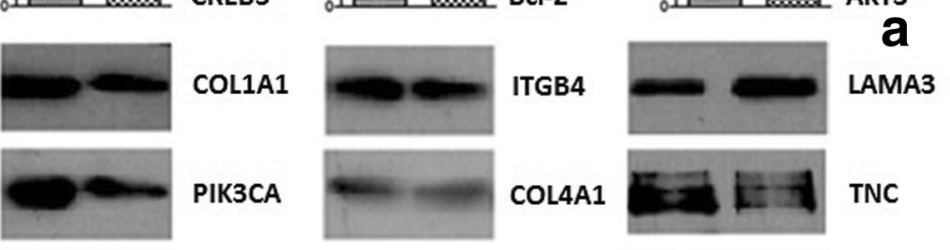

PIK3CA

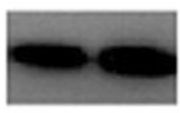

BIRC3

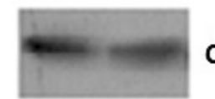

COL4A1
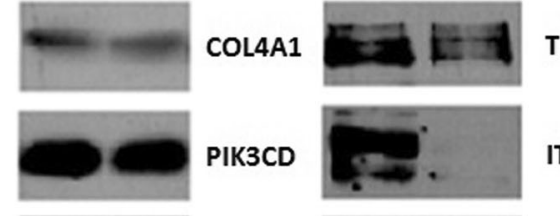

TNC

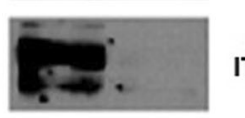

ITGA7

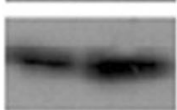

CREB5
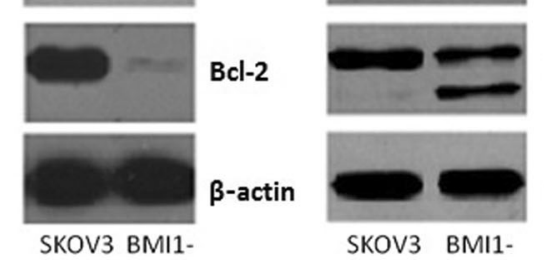

АКт3

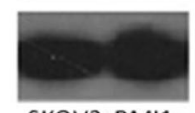

$\beta$-actin

SKOV3 BMI1-

SKOV3 BMI1-

b

Fig. 3 Validation of RNA sequencing results by $\mathbf{a}$. RT-PCR; $\mathbf{b}$. western blotting

platinum sensitivity of EOC cells. Similarly, Wang et al. revealed that down-regulating BMI1 increased the amount of reactive oxygen species, stimulated the DNA damage repair pathway, and eventually promoted the cisplatininduced apoptosis [28]. Altogether, these evidences might shed light upon the most vexing conundrum in EOC management: chemo-resistance.

The CRISPR/Cas9 technique has provided an excellent cell model for analysis of one single variable: BMI1. We further investigated the most relevant genes using RNA sequencing. Most genes differentially expressed after silencing BMI1 were engaged in tissue development, ECM organization and blood vessel development, which might alter the microenvironment and angiogenesis of tumors, and then lead to transformation of malignant phenotypes: decrease in COL1A1 and COL4A1 might redress the reconstruction disorder of ECM [29, 30]; regulation of TNC and LAMA could increase tissue tension, reduce invasion and prevent migration of ovarian cancer cells $[31,32]$. However, the clinical significance of these biomarkers in EOC management still needs validation because of controversial results from different studies. Furthermore, KEGG analyses revealed that the focal adhesion and PI3K/ AKT pathways were involved after silencing BMI1. Aberrant expression of focal adhesion kinase (FAK) was related to the uncontrolled proliferation, suppressed apoptosis, 
invasion, angiogenesis and immune-depression of tumor cells [33]. One meta-analysis demonstrated that upregulation of FAK indicated shorter overall survival in a variety of human cancers with a pooled hazards ratio of 1.815 and site specificity [34]. A growing number of studies have proved the activation of the PI3K/AKT/mTOR pathway in EOC [35-38], although the Cancer Genome Atlas research detected a low mutational rate $(<5 \%)$ of PI3KCA, AKT and PTEN in high-grade serous ovarian carcinomas ( $\mathrm{HsOCs}$ ) [39]. Other research found that PI3K genes were frequently mutated in non-HsOCs, which were relatively insensitive to platinum [38]. Another promising thing is that diverse antineoplastic drugs targeting the PI3K/AKT/mTOR pathway are under clinical investigations $[39,40]$.

\section{Conclusions}

It is feasible to use the CRISPR/Cas9 system to construct ideal EOC cell models with target gene expression thoroughly shut off. Silencing BMI1 can change various biological behaviors of EOC cells, including reducing cell proliferation, migration, invasion, and promoting cell death; BMI1 might be a promising predictor for platinum sensitivity. BMI1 may accelerate tumor genesis and metastasis through dysregulating tumor ECM and blood vessel assemblies. Molecular traits, including BMI1 and crucial genes in the focal adhesion and PI3K/ AKT pathways, might be potential biomarkers and novel therapeutic targets for EOC.

\section{Additional file}

Additional file 1: Figure S1. PARP expression detected by western blotting. (PNG $29 \mathrm{~kb}$ )

\section{Funding}

This study was supported by the National Natural Science Foundation of China (No 81402140 and No 81372780).

Availability of data and materials

Data sharing is not applicable to this article as no datasets were generated or analysed during the current study.

\begin{abstract}
Authors' contributions
Conceptualization: ZQ, GT, SK; Formal analysis: ZQ, QQ, GT; Funding acquisition: GT, SK; Investigation: ZQ, QQ; Methodology: ZQ, QQ; Project administration: GT, SK; Resources: ZQ, QQ, CD, YJ, GT, SK; Software: ZQ, QQ; Supervision: CD, YJ, SK; Validation: ZQ, CD, YJ, GT; Writing - original draft: ZQ; Writing - review \& editing: GT, CD, YJ, SK. All authors read and approved the final manuscript.
\end{abstract}

\section{Ethics approval and consent to participate}

All mice were housed and fed under specific pathogen-free conditions in an institution approved by the Beijing Laboratory Animal Research Center. All studies were approved and supervised by Peking Union Medical College Hospital.

\section{Competing interests}

The authors declare that they have no competing interests.

\section{Publisher's Note}

Springer Nature remains neutral with regard to jurisdictional claims in published maps and institutional affiliations.

\section{Author details}

'Department of Obstetrics and Gynecology, Peking Union Medical College Hospital, Chinese Academy of Medical Sciences and Peking Union Medical College, No.1 Shuaifuyuan, Dongcheng District, Beijing, China. ${ }^{2}$ Department of Gynecology and Obstetrics, West China Second University Hospital, Sichuan University, Key Laboratory of Birth Defects and Related Diseases of Women and Children (Sichuan University), Ministry of Education, Chengdu, China. ${ }^{3}$ Department of Obstetrics and Gynecology, Qilu Hospital of Shandong University, Shandong, China.

Received: 16 January 2018 Accepted: 13 April 2018

Published online: 23 April 2018

\section{References}

1. Tangjitgamol S, Manusirivithaya S, Laopaiboon M, Lumbiganon P. Interval debulking surgery for advanced epithelial ovarian cancer: a Cochrane systematic review. Gynecol Oncol. 2009;112(1):257-64.

2. Torre LA, Bray F, Siegel RL, Ferlay J, Lortet-Tieulent J, Jemal A. Global cancer statistics, 2012. CA Cancer J Clin. 2015;65(2):87-108.

3. Tanda ET, Budroni M, Cesaraccio R, Palmieri G, Palomba G, Capobianco G, et al. Epidemiology of ovarian cancer in North Sardinia, Italy, during the period 1992-2010. Eur J Gynaecol Oncol. 2015;36(1):69-72.

4. Bracken AP, Kleine-Kohlbrecher D, Dietrich N, Pasini D, Gargiulo G, Beekman $C$, et al. The Polycomb group proteins bind throughout the INK4A-ARF locus and are disassociated in senescent cells. Genes Dev. 2007;21(5):525-30.

5. Siddique HR, Saleem M. Role of BMI1, a stem cell factor, in cancer recurrence and chemoresistance: preclinical and clinical evidences. Stem Cells. 2012;30(3):372-8.

6. Song LB, Zeng MS, Liao WT, Zhang L, Mo HY, Liu WL, Set a. Bmi-1 is a novel molecular marker of nasopharyngeal carcinoma progression and immortalizes primary human nasopharyngeal epithelial cells. Cancer Res. 2006;66(12):6225-32

7. Huber GF, Albinger-Hegyi A, Soltermann A, Roessle M, Graf N, Haerle SK, et al. Expression patterns of Bmi-1 and p16 significantly correlate with overall, disease-specific, and recurrence-free survival in oropharyngeal squamous cell carcinoma. Cancer. 2011;117(20):4659-70.

8. Crea F, Duhagon Serrat MA, Hurt EM, Thomas SB, Danesi R, Farrar WL. BMI1 silencing enhances docetaxel activity and impairs antioxidant response in prostate cancer. Int J Cancer. 2011;128(8):1946-54.

9. Wang H, Liu H, Li X, Zhao J, Zhang H, Mao J, et al. Estrogen receptor a-coupled Bmi1 regulation pathway in breast cancer and its clinical implications. BMC Cancer. 2014;14:122.

10. Park IK, Morrison SJ, Clarke MF. Bmi1, stem cells, and senescence regulation. Clin Invest. 2004;113(2):175-9.

11. Engelsen IB, Mannelqvist M, Stefansson IM, Carter SL, Beroukhim R, Øyan AM, et al. Low BMI-1 expression is associated with an activated BMI-1-driven signature, vascular invasion, and hormone receptor loss in endometrial carcinoma. Br J Cancer. 2008;98(10):1662-9.

12. Gui T, Bai H, Zeng J, Zhong Z, Cao D, Cui Q, et al. Tumor heterogeneity in the recurrence of epithelial ovarian cancer demonstrated by polycomb group proteins. Onco Targets Ther. 2014;7:1705-16.

13. Hsu PD, Lander ES, Zhang F. Development and applications of CRISPR-Cas9 for genome engineering. Cell. 2014;157(6):1262-78.

14. Cho SW, Kim S, Kim JM, Kim JS. Targeted genome engineering in human cells with the Cas9 RNA-guided endonuclease. Nat Biotechnol. 2013;31(3): 230-2.

15. Ishino $Y$, Shinagawa $H$, Makino $K$, Amemura M, Nakata A. Nucleotide sequence of the iap gene, responsible for alkaline phosphatase isozyme conversion in Escherichia coli, and identification of the gene product. J Bacteriol. 1987;169(12):5429-33.

16. Mojica FJ, Díez-Villaseñor C, Soria E, Juez G. Biological significance of a family of regularly spaced repeats in the genomes of archaea, Bacteria and mitochondria. Mol Microbiol. 2000;36(1):244-6.

17. Pennisi E. The CRISPR craze. Science. 2013;341(6148):833-6.

18. Barrangou R, Fremaux C, Deveau H, Richards M, Boyaval P, Moineau S, et al. CRISPR provides acquired resistance against viruses in prokaryotes. Science. 2007;315(5819):1709-12. 
19. Morey L, Helin K. Polycomb group protein-mediated repression of transcription. Trends Biochem Sci. 2010;35(6):323-32.

20. Francis NJ, Kingston RE, Woodcock CL. Chromatin compaction by a polycomb group protein complex. Science. 2004;306(5701):1574-7.

21. Zhou W, Zhu P, Wang J, Pascual G, Ohgi KA, Lozach J, et al. Histone H2A monoubiquitination represses transcription by inhibiting RNA polymerase II transcriptional elongation. Mol Cell. 2008;29(1):69-80.

22. Abd El hafez A, El-Hadaad HA. Immunohistochemical expression and prognostic relevance of Bmi-1, a stem cell factor, in epithelial ovarian cancer. Ann Diagn Pathol. 2014;18(2):58-62.

23. Molofsky AV, He S, Bydon M, Morrison SJ, Pardal R. Bmi-1 promotes neural stem cell self-renewal and neural development but not mouse growth and survival by repressing the p161nk4a and p19Arf senescence pathways. Genes Dev. 2005;19(12):1432-7.

24. Allegra E, Trapasso S, Pisani D, Puzzo L. The role of BMI1 as a biomarker of cancer stem cells in head and neck cancer: a review. Oncology. 2014;86(4): 199-205.

25. Bhattacharya R, Nicoloso M, Arvizo R, Wang E, Cortez A, Rossi S, et al. MiR15a and MiR-16 control Bmi-1 expression in ovarian cancer. Cancer Res. 2009;69(23):9090-5.

26. Koren A, Rijavec M, Kern I, Sodja E, Korosec P, Cufer T. BMI1, ALDH1A1, and CD133 transcripts connect epithelial-mesenchymal transition to Cancer stem cells in lung carcinoma. Stem Cells Int. 2016;2016:9714315.

27. Kim BR, Kwon Y, Rho SB. BMI-1 interacts with sMEK1 and inactivates SMEK1induced apoptotic cell death. Oncol Rep. 2017;37(1):579-86.

28. Wang E, Bhattacharyya S, Szabolcs A, Rodriguez-Aguayo C, Jennings NB, Lopez-Berestein $G$, et al. Enhancing chemotherapy response with Bmi-1 silencing in ovarian cancer. PLoS One. 2011;6(3):e17918.

29. Wilson KE, Bartlett JM, Miller EP, Smyth JF, Mullen P, Miller WR, et al. Regulation and function of the extracellular matrix protein tenascin- $C$ in ovarian cancer cell lines. Br J Cancer. 1999;80(5-6):685-92.

30. Campo E, Merino MJ, Tavassoli FA, Charonis AS, Stetler-Stevenson WG Liotta LA. Evaluation of basement membrane components and the $72 \mathrm{kDa}$ type IV collagenase in serous tumors of the ovary. Am J Surg Pathol. 1992; 16(5):500-7.

31. Byers LJ, Osborne JL, Carson LF, Carter JR, Haney AF, Weinberg JB, et al. Increased levels of laminin in ascitic fluid of patients with ovarian cancer. Cancer Lett. 1995;88(1):67-72.

32. Didem T, Faruk T, Senem K, Derya D, Murat S, Murat G, et al. Clinical significance of serum tenascin-c levels in epithelial ovarian cancer. Tumour Biol. 2014;35(7):6777-82.

33. Sulzmaier FJ, Jean C, Schlaepfer DD. FAK in cancer: mechanistic findings and clinical applications. Nat Rev Cancer. 2014;14(9):598-610.

34. de Graeff P, Crijns AP, de Jong S, Boezen M, Post WJ, de Vries EG, et al. Modest effect of p53, EGFR and HER-2/neu on prognosis in epithelial ovarian cancer: a meta-analysis. Br J Cancer. 2009;101(1):149-59.

35. Levine DA, Bogomolniy F, Yee CJ, Lash A, Barakat RR, Borgen Pl, et al. Frequent mutation of the PIK3CA gene in ovarian and breast cancers. Clin Cancer Res. 2005;11(8):2875-8.

36. Bai H, Li H, Li W, Gui T, Yang J, Cao D, et al. The PI3K/AKT/mTOR pathway is a potential predictor of distinct invasive and migratory capacities in human ovarian cancer cell lines. Oncotarget. 2015;6(28):25520-32.

37. Carpten JD, Faber AL, Horn C, Donoho GP, Briggs SL, Robbins CM, et al. A transforming mutation in the pleckstrin homology domain of AKT1 in cancer. Nature. 2007:448(7152):439-44.

38. Kuo KT, Mao TL, Jones S, Veras E, Ayhan A, Wang TL, et al. Frequent activating mutations of PIK3CA in ovarian clear cell carcinoma. Am J Pathol. 2009;174(5):1597-601. 6

39. Verhaak RG, Tamayo P, Yang JY, Hubbard D, Zhang H, Creighton CJ, et al. Cancer genome atlas research network. Prognostically relevant gene signatures of high-grade serous ovarian carcinoma. J Clin Invest. 2013; 123(1):517-25.

40. Engelman JA, Chen L, Tan X, Crosby K, Guimaraes AR, Upadhyay R, et al. Effective use of PI3K and MEK inhibitors to treat mutant Kras G12D and PIK3CA H1047R murine lung cancers. Nat Med. 2008;14(12):1351-6.

\section{Ready to submit your research? Choose BMC and benefit from:}

- fast, convenient online submission

- thorough peer review by experienced researchers in your field

- rapid publication on acceptance

- support for research data, including large and complex data types

- gold Open Access which fosters wider collaboration and increased citations

- maximum visibility for your research: over $100 \mathrm{M}$ website views per year

At BMC, research is always in progress.

Learn more biomedcentral.com/submissions 